\title{
Clinicopathological Study of Cystic Lesions of Pancreas at A Tertiary Care Center
}

\author{
Vani Mundath*, Jeena Sam Kachappilly and Geetha Kizhakkethara \\ Department of Pathology, Academy of Medical Sciences, Pariyaram, Kerala, India.
}

\section{ABSTRACT}

Background: Cystic lesions of pancreas though uncommon are being increasingly identified as a result of developments in imaging techniques. Rapid advances in pancreatic surgeries associated with better patient outcome have led to a variety of pancreatic lesions being increasingly resected and pathologically diagnosed. Newer entities and classification systems have emerged.

Methods: This was a hospital based study conducted in the Department of Pathology, Academy of Medical sciences, Pariyaram. In the 91pancreatic resections done over a period of 3 years from 2013-2016, there were 15 cases of cystic pancreatic lesions which formed the subject of the present study.

Result: Cystic pancreatic lesions constituted $16.5 \%$ of cases (15 cases) of the 91 pancreatic resections done for various reasons. Of the 15 cases there were 4 non-neoplastic cysts all of which were pancreatic pseudocysts with a M:F ratio of 3:1. There were 11 neoplastic cystic lesions with 4 serous cystadenomas, 3 solid papillary pseudotumors , 2 mucinous cystadenomas and 1 case each of invasive adenocarcinoma in Intraductal Papillary Mucinous Neoplasm (IPMN) and IPMN with low grade dysplasia Except for the one patient with invasive adenocarcinoma in IPMN all others with neoplastic lesions were females. Age of the patients ranged from 14-67 yrs.

Conclusion: Cystic tumors of the pancreas are more common in females with the majority of the tumors being benign when compared to ductal adenocarcinomas which are aggressive tumors with higher incidence in males. The possibility of Solid papillary pseudotumor should always be considered in young females presenting with an encapsulated pancreatic mass especially in the tail of pancreas.

\section{Keywords: cyst, Serous Cystadenoma, Solid Papillary Pseudotumor, Pseudocyst, Whipple's Resection}

\section{Introduction}

Cystic lesions of the pancreas are rare. Pancreatic cysts may be non- neoplastic or neoplastic. Non-neoplastic cysts are classified into retention cysts and pseudocysts. ${ }^{[1]}$ Neoplastic cysts may be true cystic neoplasms or secondary cystic change in malignancies like ductal adenocarcinomas. ${ }^{[2]}$ The true nature of cystic lesions of pancreas, now being increasingly detected due to advanced imaging modalities can only be made by a thorough histopathological evaluation. When compared to the most common ductal adenocarcinoma of pancreas, Pancreatic Cystic Neoplasms (PCNs) have a favourable prognosis and many a lesions can be cured by surgical resection alone. ${ }^{[3]}$ There are a few cystic tumors of the pancreas which are classified under tumors of low malignant potential . Of these solid pseudopapillary tumor is a rare tumor reported mostly in young females. The histogenesis of this tumor is unknown. There are occasional case reports of recurrence of this tumor after surgical resection. ${ }^{[1,4]}$

The incidence of pancreatic resections for cystic lesions of pancreas is on rise. Studies on the distribution of pancreatic cystic lesions and its pathological features are few. Histopathology is the gold standard for categorizing non- neoplastic and neoplastic cystic lesions and to further grade the neoplastic lesions. Studies on cystic pancreatic lesions have not been conducted in North Kerala. Aim of the study is to categorize various cystic lesions in pancreatic resection specimens and to study the clinicopathological features of pancreatic cystic lesions.

\section{Materials and Methods}

During the period from January 2013 to December 2016, 91 patients had undergone pancreatic resection in Academy of Medical sciences, Pariyaram. There were 15 cases of cystic lesions of pancreas in the 91 pancreatic resections which formed the subject of the present study.

All resected cases were received in 10\% buffered formalin. Partial sectioning after inking of resected margin was done to aid fixation. After fixation, specimens were sliced, grossly examined and relevant areas sectioned for tissue processing. All paraffin embedded blocks obtained after processing were sectioned and stained with hematoxylin and eosin (H\&E) for histopathological examination.

Statistical analysis used: Descriptive statistics like frequency, percentage, mean and median. 


\section{Result}

Of the 91 pancreatic resections there were 66 Whipple's resections, 23 distal pancreatectomy with splenectomy,1 total pancreatectomy and 1 cystectomy. M:F ratio was 1.46 $: 1$ with age range of 14-85 years. Of these there were 24 non-neoplastic lesions, 9 benign neoplasms ,3 neoplasms of low malignant and 55 malignant neoplasms. Benign and borderline neoplasms showed female preponderance while malignant lesions showed male predominance with majority of the patients being above 60 years of age. (Table: -1)

In the 15 cystic pancreatic lesions there were 4 nonneoplastic cysts and11 neoplastic cysts. M:F ratio was 1:2.75 with age range of 14-67 years. For non-neoplastic cysts the $\mathrm{M}: \mathrm{F}$ ratio was $3: 1$ and for neoplastic cysts the M:F ratio was 1:10. (Table: -2)

In the neoplastic cyst category serous cystadenomas predominated with 3 microcystic types and 1 macrocystic type. All the 4 patients were females with age range of $42-$ 62 years. The tumor was located in the head of pancreas in a case of microcystic serous tumor. In all other cases it was in the tail of pancreas. Microcystic types showed multiple small cysts with sponge like cut surface and central scar, size ranged from 6-10cms. (Figure: -1a) Microscopy showed small cystic spaces lined by bland cuboidal cells with clear cytoplasm and sparse stroma in between the cysts (Figure: - 1b, c, d). Macrocystic type had uniloculated cyst which measured $6 \mathrm{cms}$ and microscopy showed lining by bland cuboidal epithelium.

The next frequent cystic neoplasm was solid papillary pseudotumor. All the 3 patients were females with tumors located in the tail of pancreas and age ranged from 14-40 years. All the lesions were encapsulated with solid and cystic areas, tumor size ranged from 4-10 cms.(Figure:2a) Microscopy showed solid areas with small cells showing bland nuclei, nuclear grooves, moderate amount of eosinophilic to clear cytoplasm and dyshesive tumor cells forming pseudopapillae . Foci with sheets of foamy macrophages and cholesterol clefts were identified in 2 cases. (Figure: $-2 b, c, d$ )

There were 2 mucinous cystadenomas both in females aged 43 years and 58 years and the tumors were located in the tail of pancreas. The first patient had a $13 \times 11 \mathrm{cms}$ multiloculated cyst filled with jelly like material and the second patient had a $4 \times 3 \mathrm{cms}$ biloculated cyst. Both the cases showed cyst wall lined by mucinous epithelium without atypia and subepithelial ovarian like stroma. (Figure: -3)

The single case of intestinal type main duct intraductal papillary mucinous neoplasm (MD-IPMN) with invasive adenocarcinoma was seen in a 67 year old male.Total pancreatectomy done showed marked dilatation involving the whole length of main pancreatic duct with mucinous material and multiple gray white solid papillary lesions filling the dilated duct.(Figure:-4a,b) Microscopy showed a tumor with intraductal complex papillary architecture lined by intestinal type columnar epithelium with severe dysplasia and focal areas of invasive malignancy. (Figure:-4c,d)

Branch duct IPMN with low grade dysplasia was identified inWhipple's resection specimen in a 60 year old female. Gross examination showed $2.5 \times 2 \mathrm{cms}$ mucin filled cyst in the head of pancreas and mildly dilated pancreatic ducts. Microscopy showed gastric type mucinous epithelium with focal papillary pattern and minimal atypia.

There were 4 non-neoplastic cysts all of which were pseudocysts of pancreas. M:F ratio was 3:1 and the age range was 30-45 years. Gross examination showed thick walled cyst with intraluminal brownish fluid and ragged inner surface in all the cases. Size ranged from 3-10cms. Microscopy showed fibrous cyst wall and granulation tissue without any lining epithelium. Associated chronic pancreatitis was seen in 2 cases. (Figure: - 5)

Table 1: Clinicopathological spectrum in pancreatic resections.

\begin{tabular}{|c|c|c|c|c|c|c|c|c|c|}
\hline & \multirow{2}{*}{ Diagnosis } & \multirow{2}{*}{ No.of cases } & \multicolumn{2}{|c|}{ Sex } & \multicolumn{3}{|c|}{ Age group(in years) } & \multirow[b]{2}{*}{$>60$} & \multirow{2}{*}{$\%$ of cases } \\
\hline & & & $\mathbf{M}$ & $\mathbf{F}$ & $10-20$ & $21-40$ & 41-60 & & \\
\hline I & Non-neoplastic lesions & 24 & 19 & 5 & - & 7 & 15 & 2 & 26.4 \\
\hline a & Chronic pancreatitis & 20 & 16 & 4 & - & 3 & 15 & 2 & \\
\hline b & Chronic pancreatitis with pseudocyst & 2 & 1 & 1 & - & 2 & - & - & \\
\hline c & Pseudocyst & 2 & 2 & - & & 2 & - & - & \\
\hline II & Neoplastic lesions & 67 & & & & & & & 73.6 \\
\hline 1. & Benign & 9 & 1 & 8 & - & - & 7 & 2 & 9.9 \\
\hline
\end{tabular}




\begin{tabular}{|c|c|c|c|c|c|c|c|c|c|}
\hline & \multirow{2}{*}{ Diagnosis } & \multirow{2}{*}{ No.of cases } & \multicolumn{2}{|c|}{ Sex } & \multicolumn{3}{|c|}{ Age group(in years) } & \multirow[b]{2}{*}{$>60$} & \multirow{2}{*}{$\%$ of cases } \\
\hline & & & M & $\mathbf{F}$ & $10-20$ & $21-40$ & $41-60$ & & \\
\hline a & Serous cystadenoma & 4 & - & 4 & - & - & 2 & 2 & \\
\hline b & Tubulovillous adenoma-ampulla & 2 & 1 & 1 & - & - & 2 & - & \\
\hline c & Mucinous cystadenoma & 2 & - & 2 & - & - & 2 & - & \\
\hline d & IPMN with low grade dysplasia & 1 & - & 1 & - & - & 1 & - & \\
\hline 2. & Low malignant potential & 3 & - & 3 & 1 & 2 & - & - & 3.3 \\
\hline a & Solid pseudopapillary tumor & 3 & - & 3 & 1 & 2 & & & \\
\hline 3. & Malignant & 55 & 34 & 21 & - & 2 & 21 & 32 & 60.4 \\
\hline a & Ductal adenocarcinoma & 39 & 26 & 13 & - & 2 & 16 & 21 & \\
\hline b & Neuroendocrine carcinoma & 2 & 1 & 1 & - & - & 1 & 1 & \\
\hline c & Mixed adeno-neuroendocrine carcinoma & 4 & 2 & 2 & - & - & 3 & 1 & \\
\hline d & Adenosquamous carcinoma & 2 & - & 2 & - & - & - & 2 & \\
\hline e & Ampullary carcinoma & 3 & 1 & 2 & - & - & - & 3 & \\
\hline f & IPMN with adenocarcinoma & 1 & 1 & - & - & - & - & 1 & \\
\hline g & Metastatic disease/infiltration & 4 & 3 & 1 & - & - & 1 & 3 & \\
\hline
\end{tabular}

Table 2: Clinicopathological spectrum of pancreatic cystic lesions.

\begin{tabular}{|c|c|c|c|}
\hline & Age/Sex & Type of resection & Diagnosis \\
\hline 1 & $40 / F$ & Distal pancreatectomy \&splenectomy & Solid papillary pseudotumor \\
\hline 2 & $30 / \mathrm{M}$ & Distal pancreatectomy \&splenectomy & Pseudocyst with chronic pancreatitis \\
\hline 3 & $14 / F$ & Distal pancreatectomy \&splenectomy & Solid papillary pseudotumor \\
\hline 4 & $43 / F$ & Distal pancreatectomy \&splenectomy & Mucinous cystadenoma \\
\hline 5 & $67 / M$ & Total pancreatectomy & Intestinal type MD-IPMN with invasive adenocarcionoma \\
\hline 6 & $36 / F$ & Distal pancreatectomy \&splenectomy & Solid papillary pseudotumor \\
\hline 7 & $62 / F$ & Distal pancreatectomy \&splenectomy & Microcystic seroous cystadenoma \\
\hline 8 & $42 / F$ & Whipple's resection & Microcystic seroous cystadenoma \\
\hline 9 & $62 / F$ & Distal pancreatectomy \&splenectomy & Microcystic seroous cystadenoma \\
\hline 10 & $48 / F$ & Distal pancreatectomy \&splenectomy & Macrocystic serous cystadenoma \\
\hline 11 & $39 / \mathrm{M}$ & Cystectomy & Pseudocyst \\
\hline 12 & $36 / \mathrm{M}$ & Whipple's resection & Pseudocyst with chronic pancreatitis \\
\hline 13 & $45 / F$ & Distal pancreatectomy \&splenectomy & Pseudocyst \\
\hline 14 & $60 / F$ & Whipple's resection & IPMN with low grade dysplasia \\
\hline 15 & $58 / F$ & Distal pancreatectomy \&splenectomy & Mucinous cystadenoma \\
\hline
\end{tabular}

MD-IPMN :Moderately differentiated intraductal papillary mucinous neoplasm;F-female;M-Male. 


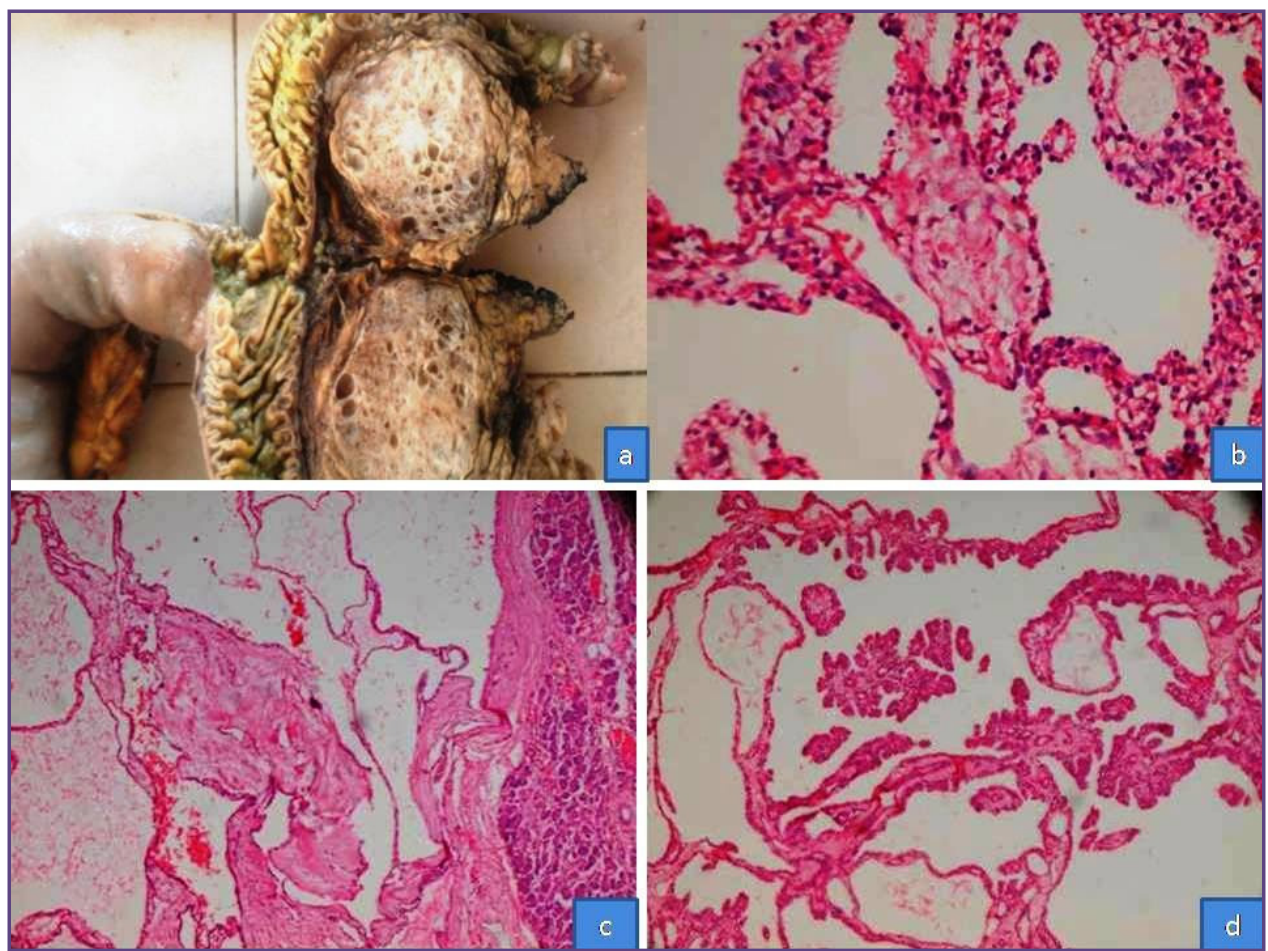

Fig. 1: Microcystic serous cystadenoma :a-Whipple's resection specimen with a circumscribed tumor of sponge like consistency with microcysts in head of pancreas; b,c\&d- Photomicrograph showing small cystic spaces lined by bland cuboidal cells with clear cytoplasm (H\&E-10x).
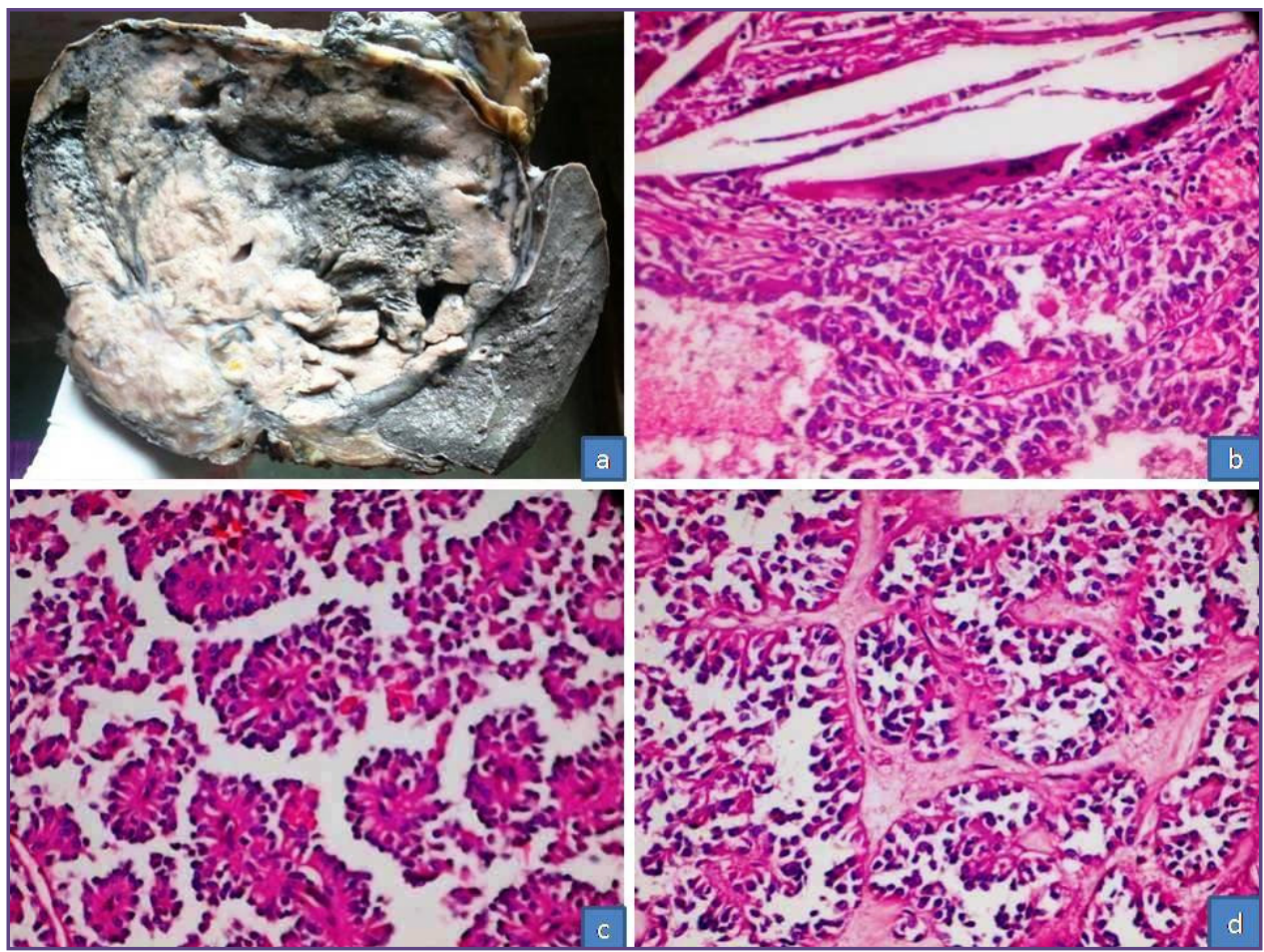

Fig. 2: Solid Pseudopapillary Tumor- a)Distal pancreatectomy with splenectomy,encapsulated tumor with solid and cystic areas in tail of pancreas;b,c \& d)Photomicrograph showing bland,dyshesive cells forming pseudopapillae.Cholesterol clefts and collections of foam cells noted in $b(H \& E-40 x)$. 


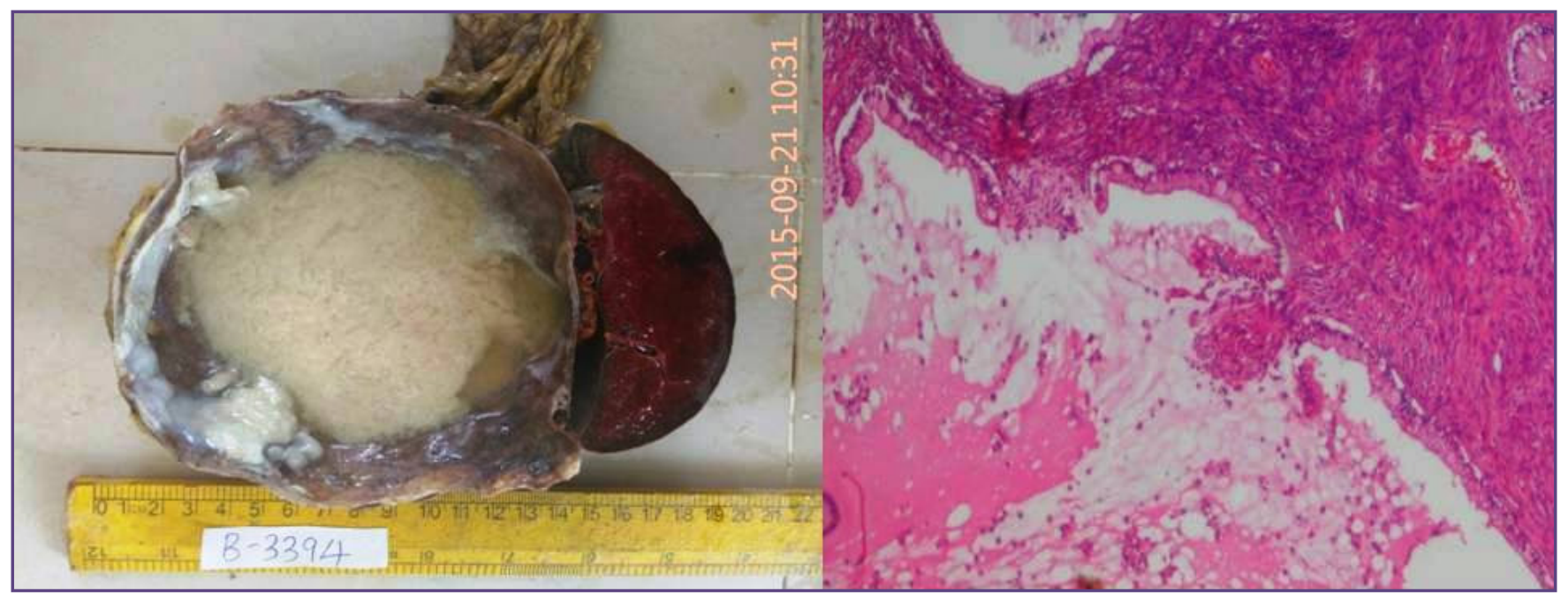

Fig. 3: mucinous cystadenoma. Gross- Distal pancreatectomy with splenectomy, multiloculated cyst with jelly like material in tail of pancreas .Photomicrograph- cyst lined by mucinous epithelium without atypia and subepithelial ovarian like stroma.(H\&E-40x).
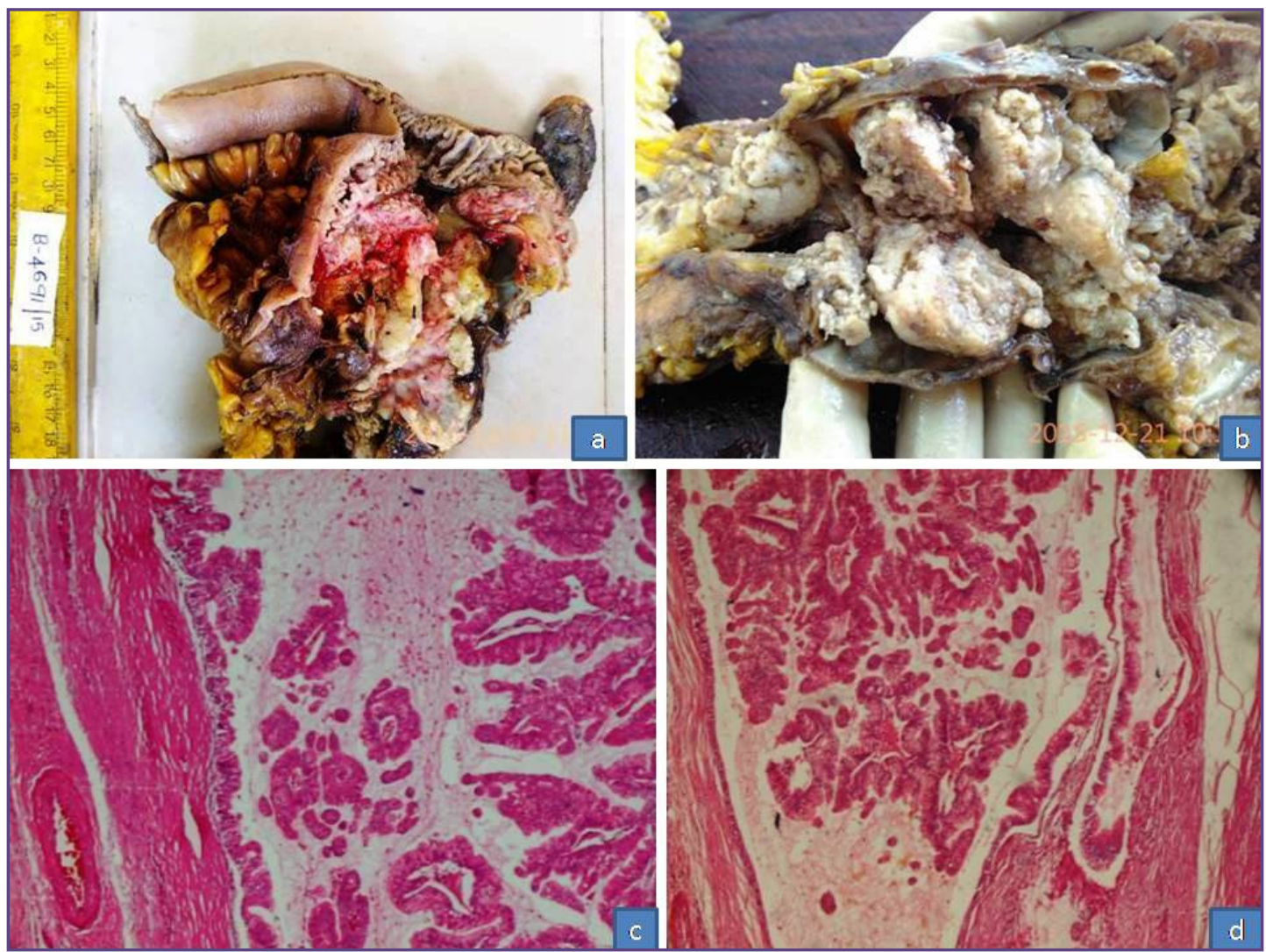

Fig. 4: Invavive adenocarcinoma in IPMN .a\&b)Total pancreatectomy - markedly dilated main duct, mucinous material and multiple gray white solid papillary lesions filling the dilated duct;c\&d)photomicrograph showing intraductal complex papillary architecture lined by intestinal type columnar epithelium with severe dysplasia and areas of invasive malignancy. (H\&E-40x). 

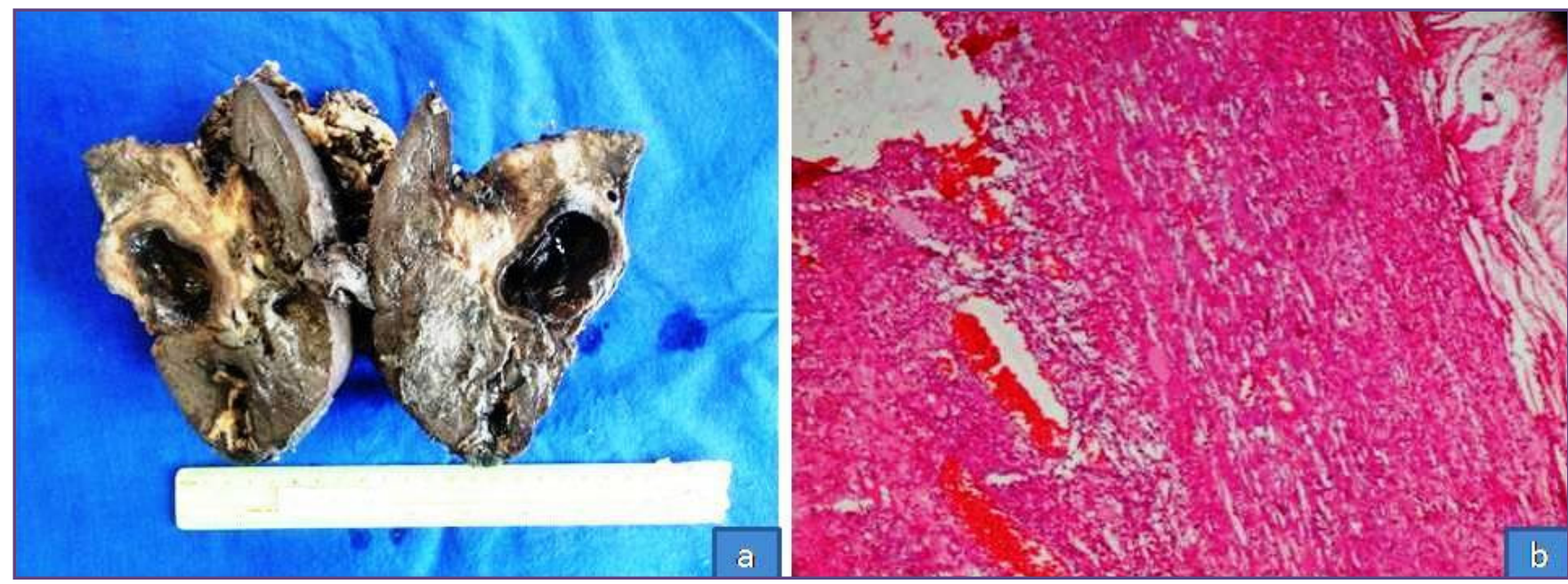

Fig. 5: Pancreatic pseudocyst.a(gross)- Distal pancreatectomy with splenectomy ,thick walled cyst in tail of pancreas. b(photomicrograph) - fibrous cyst wall and granulation tissue without any lining epithelium.(H\&E-40x).

\section{Discussion}

A variety of non- neoplastic, benign and malignant cystic lesions can occur in the pancreas. Pathologically, pancreatic cystic lesions are classified into simple retention cysts, pseudocysts and cystic neoplasms. The non-neoplastic cysts are further divided into epithelial cysts and nonepithelial or pseudocysts. Non-neoplastic pancreatic cysts may mimic neoplastic cysts on radiology. ${ }^{[1]}$ So such cysts may be encountered in pathological practice, the nonneoplastic nature of which is confirmed by histopathology. Pseudocysts are the most common non-neoplastic cysts of the pancreas and have a strong association with chronic pancreatitis. Other less frequently encountered nonneoplastic cysts include lymphoepithelial cysts, epidermoid cysts and infectious cysts. ${ }^{[6]}$

In contrast with solid tumors of pancreas, most of which are ductal adenocarcinomas with grave outcomes, the vast majority of cystic neoplasias are either benign tumors or low-grade malignancies with indolent behaviour. ${ }^{[7]}$ Cystic tumors constitute $5-10 \%$ of all pancreatic neoplasms. Most common true cystic neoplasms of the pancreas are serous cystic neoplasms. Mucinous cystic neoplasms (MCN) are the second commonest and is seen almost exclusively in perimenopausal females. Invasive carcinoma may develop in MCNs. ${ }^{[8]}$ So extensive sampling is warranted. Other rare true cystic neoplasms are intraductal papillary mucinous neoplasms and solid papillary pseudotumors.

Cystic change can rarely be seen in ductal adenocarcinoma which is the commonest pancreatic tumor. It may be due to central necrosis, cystic dilatation of distally located obstructed ducts or due to cystic dilatation of infiltrating tubular units. ${ }^{[2]}$
Serous cystadenomas are benign tumors. Because of the presence of abundant intracytoplasmic glycogen rendering clear cell morphology to the lining cells they are also known as glycogen rich cystadenomas. ${ }^{[9]}$ Patients may present with abdominal pain or with incidentally detected lesion on radiological evaluation. Tumors can occur anywhere in the pancreas with a predilection for body and tail. Grossly the tumors are well circumscribed with central scar and spongy cut surface. Microscopy show multiple cystic spaces lined by cuboidal cells with clear cytoplasm. ${ }^{[9,10,11]}$

Serous cystadenocarcinomas are extremely rare tumors with less than 20 cases reported so far. The gross features and cytoarchitecture of this tumor is indistinguishable from serous cystadenomas. In the one case reported by Van Dyke et al patient had liver metastasis at the time of resection. The resected tumor was well circumscribed, measured $15.5 \times 12 \mathrm{cms}$ and on microscopy demonstrated the typical cytoarchitecture of serous cystadenoma except for rare foci of perineural invasion. There is no definite histopathological criteria to diagnose cystadenocarcinoma though vascular and perineural invasion and direct invasion into adjacent structures indicates a locally aggressive disease and malignant potential. The diagnosis of malignancy can only be established by the clinical behaviour of the neoplasm (metastatic disease). All cystadenocarcinomas reported are $>4 \mathrm{cms}$ in diameter. So serous cystic tumors more than $4 \mathrm{cms}$ should be considered for resection. ${ }^{[12]}$

Mucinous cystic neoplasms of the pancreas almost exclusively occur in women predominantly in the body and the tail of pancreas, with peak incidence in fifth decade.[8] MCNs are defined by the World Health Organization (WHO) as cystic epithelial neoplasms that 
occur almost exclusively in women; do not communicate with the pancreatic ductal system and which are composed of columnar, mucin-producing epithelium, supported by ovarian-type stroma. These tumors are classified into MCN with mild dysplasia (adenoma), with moderate dysplasia (borderline neoplasm), with high grade dysplasia and $\mathrm{MCN}$ with invasive carcinoma. The prevalence of invasive cancer in $\mathrm{MCN}$ is low. ${ }^{[13]} \mathrm{MCN}$ have low biologic aggressiveness and the prognosis is much better than that of ductal carcinoma. ${ }^{[14]}$

Intraductal Papillary Mucinous Neoplasms (IPMN) are cystic tumors of the pancreas and occur mainly in elderly males. It may involve the main duct (MD) or the branch duct (BD) of the pancreas, produce mucin and show papillary differentiation. [15] According to the site of involvement it is classified into MD, BD and combined types. MD-IPMN show segmental or diffuse dilatation of MPD $>5 \mathrm{~mm}$ in the absence of other causes of obstruction. ${ }^{[16]}$

MD-IPMNs when compared to BD-IPMNs have higher malignant potential. WHO classifies IPMN into those with low grade dysplasia, intermediate grade dysplasia and high grade dysplasia. Four histological types have been identified-the gastric, intestinal, pancreaticobiliary and oncocytic type. Intestinal type IPMNs are usually MD type lesions and gastric type IPMNs are usually BD type lesions. ${ }^{[17]}$ Intestinal type lesions have a higher histological grade. As per International consensus guidelines 2012 the type of invasive carcinoma developing in IPMN, colloid versus tubular should be mentioned in reporting as it has prognostic implications. Colloid carcinomas show intestinal differentiation (CDX2 \&MUC2 positive) and have better prognosis. The gastric type is low grade (MUC5AC positive, MUC1 negative) and only a small percentage progress to invasive malignancy which is of tubular type. Oncocytic type show complex papillae lined by1-5 layers of cuboidal cells. One distinctive feature of this histological type is the presence of intraepithelial lumina containing mucin giving the tumor a cribriform architecture. Pancreaticobiliary type is considered to be a high grade version of gastric type and may progress to tubular carcinoma. Tumors may express MUC1 a marker for aggressive behaviour in pancreas. ${ }^{[18]}$

Differential diagnoses of IPMN include MCN, retention cyst and Pancreatic intraepithelial neoplasia (PaIN). MCNs do not communicate with the pancreatic duct and have subepithelial ovarian like stroma. PaINs are microscopic lesions not visible on gross examination. ${ }^{[19]}$ Schmidt CM et al recommended surgery for all fit patients with MDIPMN and for BD-IPMN with mural nodularity or positive cytology irrespective of size or location to lower the risk of invasive carcinoma. ${ }^{[20]}$
Solid papillary pseudotumor (SPPT) is a rare tumor usually seen in young females and frequently in the tail of pancreas though it can occur anywhere in the pancreas. The histogenesis of this tumor is unknown and lack definite endocrine or exocrine differentiation. [4] Tumors are usually encapsulated with solid, cystic and hemorrhagic areas. They are tumors of low grade malignant potential and complete surgical excision is the treatment of choice with excellent prognosis. There are a few case reports of local recurrence and distant metastasis in SPPT. Diagnosis of SPPT should be considered in young females with pancreatic mass. ${ }^{[21,22]}$

Other rare cystic tumor of pancreas is cystic acinar cell tumor which includes acinic cell cystadenoma and acinic cell cystadenocrcinoma. Prognosis is same as that for reglular acinic cell tumor. ${ }^{[23]}$

\section{Conclusion}

Cystic tumors of the pancreas are significantly less frequent than solid tumors. Though the incidence of non-neoplastic lesions like chronic pancreatitis and malignancies like ductal carcinoma of pancreas show male predominance cystic tumors of pancreas have a very high female preponderance. Majority of PCNs are benign. Solid papillary pseudotumors present at a relatively younger age when compared to other PCNs. In females presenting with cystic pancreatic mass on radiological examination the possibility of cystic tumors of pancreas should always be kept in the differential diagnosis as these tumors have a very favourable outcome and surgical resection is curative in most of the cases.

\section{Reference}

1. Karoumpalis I, Christodoulou DK. Cystic lesions of pancreas. Ann Gastroenterol 2016;29:155-161.

2. Odze RD, Goldbum JR. Surgical pathology of GI tract, liver, biliary tract and pancreas(3rd ed) Elseiver Saunders;2015:1093-1108.

3. Adsay NV. Cystic lesions of Pancreas. Modern Pathology 2007;20:S71-S93.

4. Vassos N, Agaimy A, Klein P, Hohenberger W, Croner RS. Solid-pseudopapillary neoplasm (SPN) of the pancreas: Case series and literature review on an enigmatic entity. Int J Clin Exp Pathol 2013;6:1051-9.

5. Hutchins G F, Draganov P V. Cystic neoplasms of the pancreas: A diagnostic challenge. World J Gastroenterol 2009 ; 15: 48-54.

6. Kim YS, Cho JH. Rare Non-neoplastic cysts of pancreas. Clin Endosc 2015;48:31-38.

7. Basturk O, Coban I, Adsay NV. Pancreatic cyst-Pathologic classification, Differential diagnosis and clinical implications. Arch Pathol Lab Med 2009;133:423-438. 
8. Brugge WR. Diagnosis and management of cystic lesions of the pancreas. J Gastrointest Oncol 2015;6:375-388.

9. Jacob S, Rawat P, Mark RP .Serous microcystic adenoma (glycogen rich cystadenoma) of the pancreas. IJPM 2010;53:106-108.

10. Omeroglu A, Paner G P, Ciesla MC, Hartman G. Serous Microcystic Adenoma of the Pancreas. Arch Pathol Lab Med 2001; 125: 1613-1614.

11. Reid MD, Choi H, Balci S, Akkas G, Adsay V.Serous cystic neoplasms of the pancreas: Clinicopahologic and molecular characteristics.Seminars in Diag Pathol 2014;31:475-483.

12. Dyke TJV, Johlin FC, Bellizzi AM, Howe JR. Serous Cystadenocarcinoma of the Pancreas: Clinical Features and Management of a Rare Tumor. Dig Surg 2016;33:240-248.

13. Sarr MG, Carpenter HA, Prabhakar LW, Orchard TF, Hughes S, van Heerden JA et al. Clinical and Pathologic Correlation of 84 Mucinous Cystic Neoplasms of the Pancreas.Can One Reliably Differentiate Benign From Malignant(or Premalignant) Neoplasms?.Ann Of Surgery2000;231: 205-212.

14. Crippa S, Salvia R, Warshaw AL, Domínguez I, Bass C, Falconi $\mathrm{M}$ et al.Mucinous Cystic Neoplasm of the Pancreas is Not an Aggressive Entity:Lessons From 163 Resected Patients. Ann Surg 2008 ; 247: 571-579.

15. Grutzmann R, Post S, Saeger HD, Niedergethmann M. Intraductal papillary mucinous neoplasia of the pancreas. Dtsch Arztebl Int 2011;108:788-794.

16. Jana T, Shroff J, Bhutani M S. Pancreatic cystic neoplasms:Reviwew of current knowledge, diagnostic challenges and management options.J Carcinog 2015;14.3.

17. Machado NO, Al Qadhi H, Al Wahibi K. Intraductal Papillary Mucinous Neoplasm of Pancreas.N Am J Med Sci 2015;7:160-175.

18. Tanaka M, Castillo CF, Adsay V, Chari S, Falconi M, Jang J-Y et al. International consensus guidelines 2012 for the management of IPMN and MCN of the pancreas. Pancreatology2012;12:183-197.

19. Castellano-Megias VM, Ibarrola-de Andres C, LopezAlonso G, Colina-Ruizdelgado F. Pathological features and diagnosis of intraductal papillary mucinous neoplasm of the pancreas.World J Gastrointest Oncol 2014;6:311-324.

20. Schmidt CM, White P, Waters JA, Yiannoutsos C, Cummings OW, Baker MS . Intraductal papillary mucinous neoplasms: predictors of malignant and invasive pathology. Ann Surg. 2007; 246:644-651.

21. Patil TB, Shrikhande SV, Kanhere HA, Saoji RR, Ramadwar MR, Shukla PJ. Solid pseudopapillary neoplasm of the pancreas: a single institution experience of 14 cases. HPB 2006; 8: 148-150.

22. Ozguven BY, Tuncel D, Polat N, Sakiz D, Kabukcuoglu F, Koksal H et al.Solid-pseudopapillary neoplasm of the pancreas: Clinicopathologic and immunohistochemical analysis of nine cases. I J P M $2015 ; 58: 292-295$.

23. Chatelain D, Paye F, Mourra N, Scoazee J-Y, Baudrimont $\mathrm{M}$, Parc R et al.Unilocular Acinar Cell Cystadenoma of the Pancreas-An Unusual Acinar Cell Tumor.Am J Clin Pathol 2002;118:211-214.

*Corresponding author:

Dr. Vani Mundath, Associate Professor, Department of Pathology, Academy of Medical Sciences, Pariyaram, Pariyaram Medical College Post.Kerala, India. Pin: 670503

Phone: +919400694097

Email: vanim250180@gmail.com

Financial or other Competing Interests: None. 